\title{
The chiral transition of $N_{f}=2$ QCD with fundamental and adjoint fermions
}

\author{
Jürgen Engels ${ }^{* \dagger}$, Sven Holtmann, and Thomas Schulze \\ Fakultät für Physik, Universität Bielefeld, D-33615 Bielefeld, Germany \\ E-mail: engelsephysik.uni-bielefeld.de, sven.holtmannedepfa.com \\ tschulze1973@gmx.de
}

\begin{abstract}
We study QCD with two staggered Dirac fermions both in the fundamental $(Q C D)$ and the adjoint representation $(a Q C D)$ near the chiral transition. The aim is to find the universality class of the chiral transition and to verify Goldstone effects below the transition. We investigate $a Q C D$, because in that theory the deconfinement and the chiral transitions occur at different temperatures $T_{d}<T_{c}$. Here, we show that the scaling behaviour of the chiral condensate in the vicinity of $\beta_{c}$ is in full agreeement with that of the $3 d O(2)$ universality class. In the region $T_{d}<T<T_{c}$ we confirm the quark mass dependence of the chiral condensate which is expected due to the existence of Goldstone modes like in $3 d O(N)$ spin models. For fundamental $Q C D$ we use the $p$ 4-action. Here, we find Goldstone effects below $T_{c}$ like in $a Q C D$ and the $3 d O(N)$ spin models, however no $O(2) / O(4)$ scaling near the chiral transition point. The result for $Q C D$ may be a consequence of the coincidence of the deconfinement transition with the chiral transition.
\end{abstract}

XXIIIrd International Symposium on Lattice Field Theory

25-30 July 2005

Trinity College, Dublin, Ireland

\footnotetext{
* Speaker.

${ }^{\dagger}$ We thank Frithjof Karsch and Martin Lütgemeier for many discussions and the use of their programs and data. We are grateful for the help of Doug Toussaint with the MILC code.
} 


\section{Introduction}

We study $Q C D$ with two staggered Dirac fermions both in the fundamental $(Q C D)$ and the adjoint representation $(a Q C D)$ near the chiral transition. The intention is to find the universality class of the transition and to verify Goldstone effects below the transition. For ordinary $Q C D$ with staggered fermions the prediction of the three-dimensional $O(2)$ universality class (the $O(4)$ class in the continuum theory) could up to now not be confirmed. We investigate in addition $a Q C D$, because in that theory the deconfinement and the chiral transitions occur at different temperatures with $T_{d}<T_{c}$ (that is $\beta_{d}<\beta_{c}$ ) [1]. The chiral transition can therefore be studied without interference. The comparison of $Q C D$ data with the critical behaviour of $O(N)$ spin models requires the following identifications of $Q C D$ variables to $O(N)$ variables: the chiral condensate corresponds to the magnetization $M$ and the quark mass $\left(m_{q} a\right)$ to the magnetic field $H$. Instead of the temperature we use $\beta=6 / g^{2}$.

\section{2. $Q C D$ with adjoint fermions $(a Q C D)$}

The action of $a Q C D$ which we use is [1]

$$
S=\beta S_{G}\left(U^{(3)}\right)+\sum_{x, y} \bar{\psi}_{x} M\left(U^{(8)}\right)_{x, y} \psi_{y}
$$

Here, the gluon part $S_{G}\left(U^{(3)}\right)$ is the usual Wilson one-plaquette action, but the fermions are in the 8-dimensional adjoint representation of color $S U$ (3). The standard staggered fermion matrix $M$ depends correspondingly on $U^{(8)}$ instead of $U^{(3)}$. The links $U^{(8)}$ are real because

$$
U_{a b}^{(8)}=\frac{1}{2} \operatorname{tr}_{3}\left[\lambda_{a} U^{(3)} \lambda_{b} U^{(3) \dagger}\right] .
$$

The fermion action does not break $Z(3)$ center symmetry and the Polyakov loop $L_{3}$ is therefore order parameter for the deconfinement transition. In the continuum $a Q C D$ contains an $S U\left(2 N_{f}\right)$ chiral symmetry which breaks to $S O\left(2 N_{f}\right)$ for $T<T_{c}$. This is because here $N_{f}$ Dirac fermions correspond to $2 N_{f}$ Majorana fermions. For $N_{f}=2$ we have $S U(4)$-symmetry which breaks to $S O(4)$. The corresponding continuum transition has been studied in Ref. [2] with renormalizationgroup methods. On the lattice an $O(2)$-symmetry remains for staggered fermions.

Our simulations [3] were done on $N_{\sigma} \times 4$ lattices with $N_{\sigma}=8,12$ and 16 and a fixed length $\tau=0.25$ of the trajectories. We used 900-2000 trajectories for measuring the chiral condensate $\langle\bar{\psi} \psi\rangle$, the Polyakov loop $L_{3}$ and the disconnected part $\chi_{\text {dis }}$ of the susceptibility.

In Ref. [1] the deconfinement transition point was found at $\beta_{d}=5.236(3)$ - the same value as in $Q C D(!)$, but here it is of first order. The usual strategy to locate the chiral transition point $\beta_{c}$ is to extrapolate the line of peak positions $\beta_{p c}$ of the susceptibility to $m_{q} a=0$ with

$$
\beta_{p c}=\beta_{c}+c\left(m_{q} a\right)^{1 / \Delta},
$$

where $\Delta=\beta_{m} \delta$ is a product of critical exponents. On the $8^{3} \times 4$ lattice we find for $m_{q} a=$ $0.005,0.01,0.02$ the values $\beta_{p c}=5.73(8), 5.74(6), 5.75(10)$. With $\Delta$ from $O(2)$ we obtain $\beta_{c}=$ 5.7(2). A better method [B] is to expand the scaling ansatz $\langle\bar{\psi} \psi\rangle=m^{1 / \delta} f(z)$ at at small $|z|$. Here, 


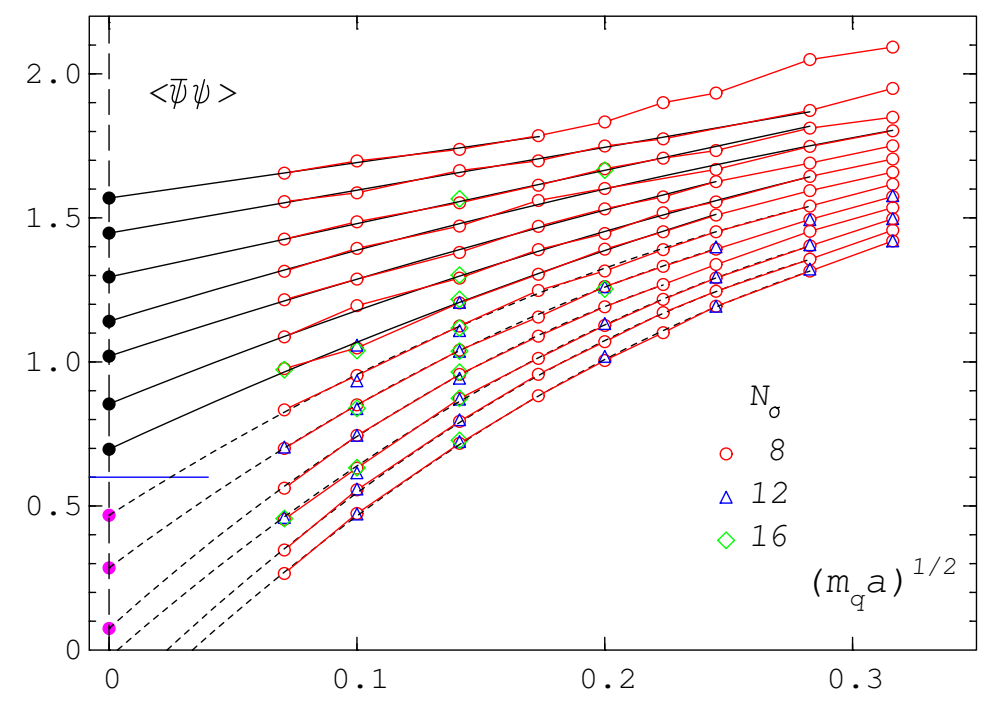

Figure 1: The chiral condensate $\langle\bar{\psi} \psi\rangle$ of $a Q C D$ as a function of $\left(m_{q} a\right)^{1 / 2}$ for all $\beta$-values between 5.3 (highest values) and 5.9 (lowest values) from $N_{\sigma}^{3} \times 4$ lattices with $N_{\sigma}=8$ (circles), 12 (triangles) and 16 (diamonds). The black lines are fits with ansatz (2.5), the filled circles denote the extrapolations to $m_{q} a=0$.

$m=m_{q} a /\left(m_{q} a\right)_{0}, \beta_{r}=\left(\beta-\beta_{c}\right) / \beta_{0}$ are the reduced field and temperature and $z=\beta_{r} m^{-1 / \Delta}$. The result is

$$
\langle\bar{\psi} \psi\rangle=\left(m_{q} a\right)^{1 / \delta}\left\{d_{c}+d_{c}^{1}\left(\beta-\beta_{c}\right)\left(m_{q} a\right)^{-1 / \Delta}+\ldots\right\} .
$$

Fits to only the first term at fixed $\beta$ are best for $\beta \in[5.6,5.7]$. If $O(2)$ exponents and two terms are used one finds a unique zero of the parameter $d_{c}^{1}\left(\beta-\beta_{c}\right)$ as a function of $\beta$ at the rather precise value $\beta_{c}=5.624(2)$.

Due to the existence of massless Goldstone modes for all $T<T_{c}$ the susceptibility $\chi_{L}$ of $3 d$ $O(N)$ spin models diverges for $H \rightarrow 0$ as $\chi_{L} \sim H^{-1 / 2}$. If $a Q C D$ behaves effectively as such a model, a similar divergence is expected. In terms of the chiral condensate we must have then

$$
\langle\bar{\psi} \psi\rangle\left(\beta, m_{q} a\right)=\langle\bar{\psi} \psi\rangle(\beta, 0)+c_{1}(\beta)\left(m_{q} a\right)^{1 / 2}+c_{2}(\beta)\left(m_{q} a\right)+\ldots
$$

Corresponding fits are shown in Fig. 1 for $\beta=5.3$ to $\beta=5.9$ with $\Delta \beta=0.05$. The blue line separates fits above and below $\beta_{c}$. We see that the model expectations are met well by the data.

We have performed explicit scaling tests for the data from the $8^{3} \times 4$ lattice (they coincide with the results from the $N_{\sigma}=12$ and 16 lattices). In the left part of Fig. 2 the data for $\langle\bar{\psi} \psi\rangle m^{-1 / \delta}$ are shown as a function of the scaling variable $z=\beta_{r} / m^{1 / \Delta}$ using $O(2)$ exponents. The normalizations $\left(m_{q} a\right)_{0}$ and $\beta_{0}$ have been determined from the behaviour at $\beta_{c}$ (2.4), and the extrapolations $\langle\bar{\psi} \psi\rangle(\beta, 0)$ obtained from the Goldstone effect [3]. In addition we show the $O(2)$ scaling function. We observe that the data scale very well with $O(2)$ exponents in the small $|z|$-region and agree there with the $O(2)$ scaling function. For decreasing $z<0$ outside the shown range the data for different $\beta$ start to deviate and exhibit corrections-to-scaling as in the original $O(2)$ model. In the right part of Fig. 2 we show the best scaling result for the unnormalized variables obtained by varying the exponents around $\delta \approx 4.0, v \approx 1.1$, the expected continuum class values [2]. Here, $\beta_{c}=5.64$. We see from Fig. $⿴$ that the data still spread considerably, a scaling function is not known up to now. 

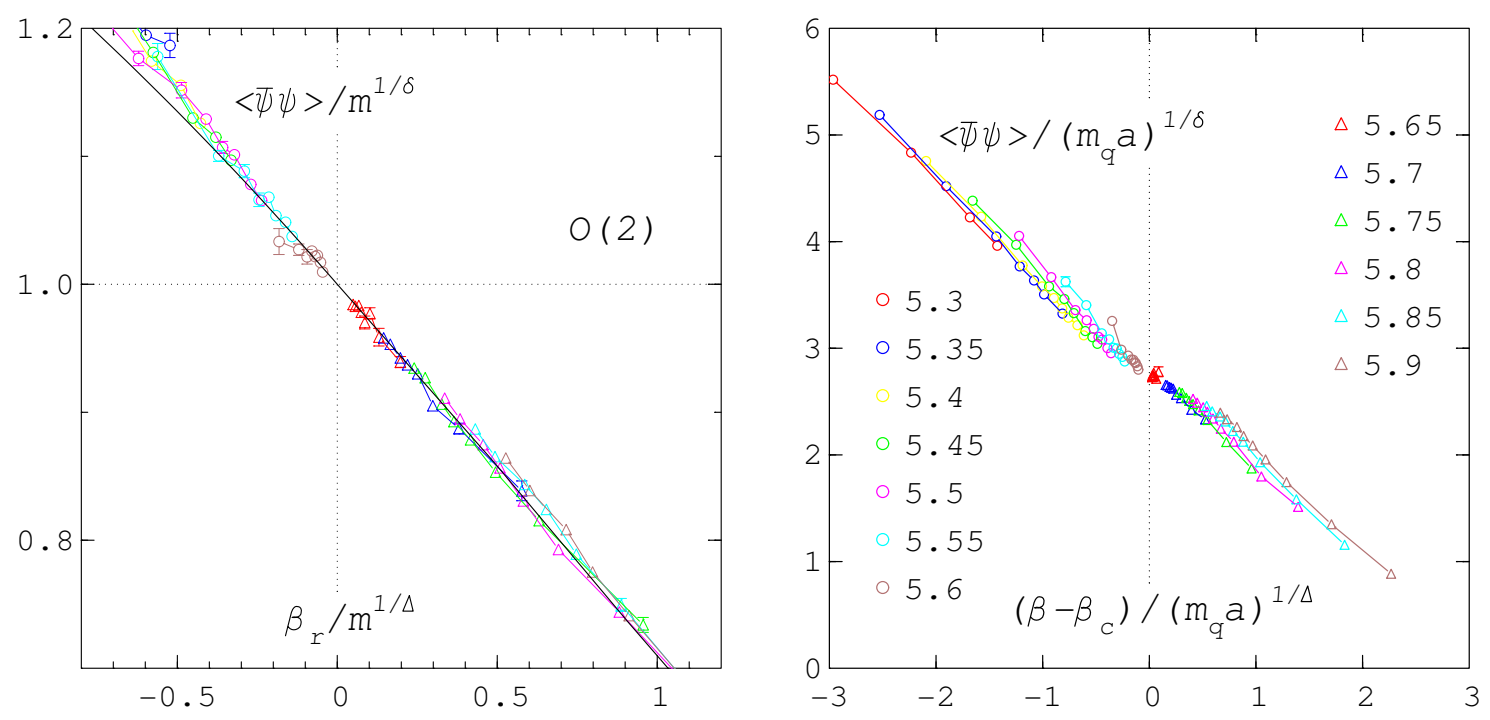

Figure 2: Scaling test in $a Q C D$. Left part: $\langle\bar{\psi} \psi\rangle / m^{1 / \delta}$ for $O(2)$ parameters in the vicinity of the critical point. The black line is the $O(2)$ scaling function. Right part: the unnormalized variables with $\delta=4.4, v=$ 1.0 from Ref. [2]. The data for fixed $\beta$-values are connected by straight lines to guide the eye.

\section{QCD with fundamental fermions}

Since the predictions for the universality class of ordinary $Q C D$ could not be confirmed with the standard staggered action we use here the $p 4$-action[ [ 4 , which improves the cut-off dependence, rotational invariance and flavour symmetry. The thermodynamics of two flavour $Q C D$ has been investigated with this action in Ref. [5]. In particular, the chiral transition point was estimated to $\beta_{c}=3.48(3)$ by extrapolation of the pseudocritical points $\beta_{p c}\left(m_{q} a\right)$ to $m_{q} a=0$.

We have extended the work of Karsch et al.[5] on $N_{\sigma}^{3} \times 4$ lattices with $N_{\sigma}=8,12,16$ at the couplings $\beta=2.8,3.0,3.2,, 3.4,3.48$ and 3.50, that is for $\beta \leq \beta_{c}$. All our simulations were done with the MILC code using the R-algorithm with a mass-dependent stepsize $\delta \tau\left(m_{q} a\right)=$ $\min \left\{0.4 m_{q} a, 0.1\right\}$ and trajectory length $\tau=1$. We produced 1000-2000 trajectories for masses $m_{q} a \in[0.025,0.5]$ and somewhat less for $m_{q} a=0.01$.

Like in $a Q C D$ we have tested the mass dependence of the chiral condensate at fixed $\beta<\beta_{c}$. In Fig. 3 we show the data from $\beta=2.8$ to $\beta=3.48$ as a function of $\left(m_{q} a\right)^{1 / 2}$. We have fitted the data in the range $m_{q} a \lesssim 0.15$ to the ansatz

$$
\langle\bar{\psi} \psi\rangle\left(\beta, m_{q} a\right)=\langle\bar{\psi} \psi\rangle(\beta, 0)+c_{1}(\beta)\left(m_{q} a\right)^{1 / 2} .
$$

Again, the behaviour expected due to the Goldstone effect is confirmed by the data.

In Fig. 4 we investigate the scaling form $\langle\bar{\psi} \psi\rangle=d_{c}\left(m_{q} a\right)^{1 / \delta}$ for the critical point in the close neighbourhood of $\beta_{c}$. We have plotted the data with $\delta_{O(2)}=4.78$ in the left part of Fig. $\theta$ at $\beta=3.48$ (solid lines) and $\beta=3.5$ (dotted lines). Obviously, the curves are no straight lines going through the origin. Also, the corresponding attempt to obtain a scaling function with $O(2)$ exponents fails as can be seen in the left part of Fig. 5. Here, $\beta_{c}=3.51$ was used, the scales are not normalized. Other values for $\beta_{c}$ shift the results slightly, but do not lead to scaling. The use of $O(4)$ exponents does not improve the result. 


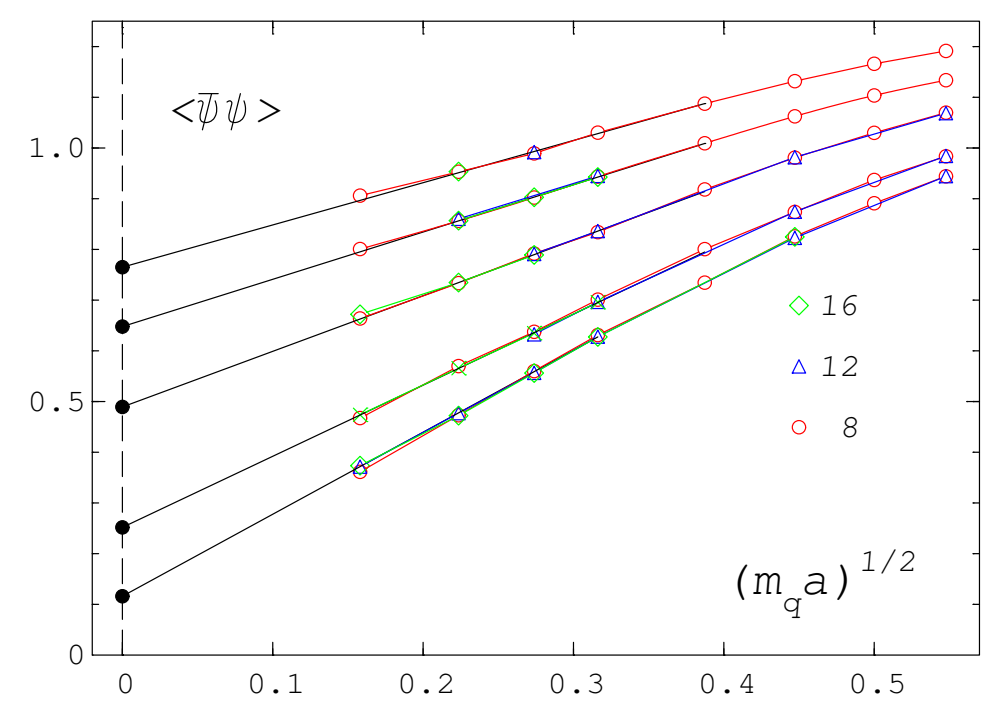

Figure 3: The chiral condensate $\langle\bar{\psi} \psi\rangle$ as a function of $\left(m_{q} a\right)^{1 / 2}$ for all $\beta$-values between 2.8 (highest values) and 3.48 (lowest values) from $N_{\sigma}^{3} \times 4$ lattices with $N_{\sigma}=8$ (circles), 12 (triangles) and 16 (diamonds). The black lines show the fit results and the filled circles the estimates for the chiral condensate at $m_{q} a=0$.

In order to achieve scaling of the data we have fitted the ansatz $\langle\bar{\psi} \psi\rangle=d_{c}\left(m_{q} a\right)^{1 / \delta}$ at $\beta=3.50$ with a free amplitude $d_{c}$ and a free exponent $\delta$ for $m_{q} a \in[0.01,0.1]$ and obtain $\delta=2.3$. The fit is shown in the right part of Fig. 4. Likewise, we estimated the magnetic exponent $\beta_{m}$ to 0.6 from the values of $\langle\bar{\psi} \psi\rangle$ at $m_{q} a=0$ which we gained from the Goldstone extrapolations. In the right part of Fig. 5 we show the data for these exponents. Here, $\langle\bar{\psi} \psi\rangle /\left(m_{q} a\right)^{1 / \delta}$ is normalized to 1 at $\beta_{c}$. Still, scaling is not perfect, but certainly much better than for $O(N)$ exponents.
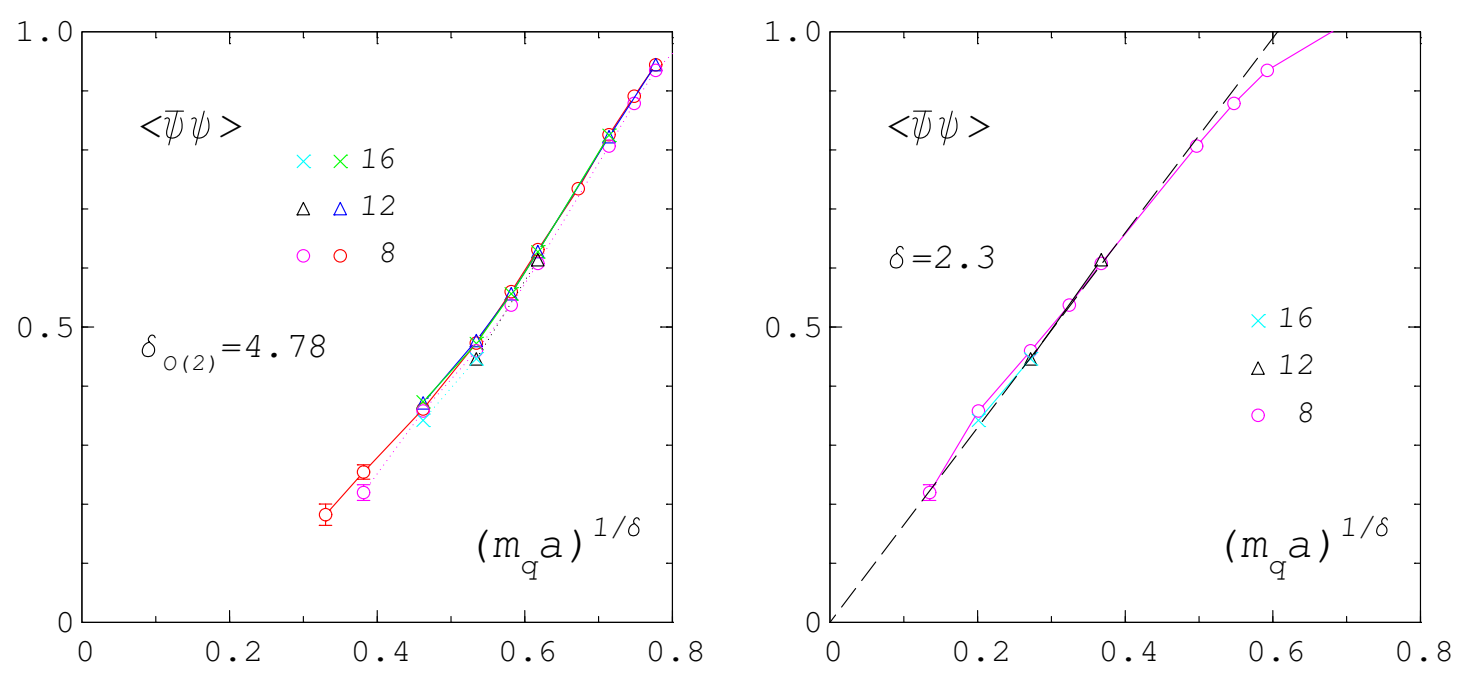

Figure 4: Test of the scaling ansatz for $\langle\bar{\psi} \psi\rangle$ at the critical point for $O(2)$ (left part) at $\beta=3.48$ (solid lines) and 3.5 (dotted lines). In the right part we show a free fit to the scaling ansatz at $\beta=3.5$ (dashed line). Here, the exponent is $\delta=2.3$. 

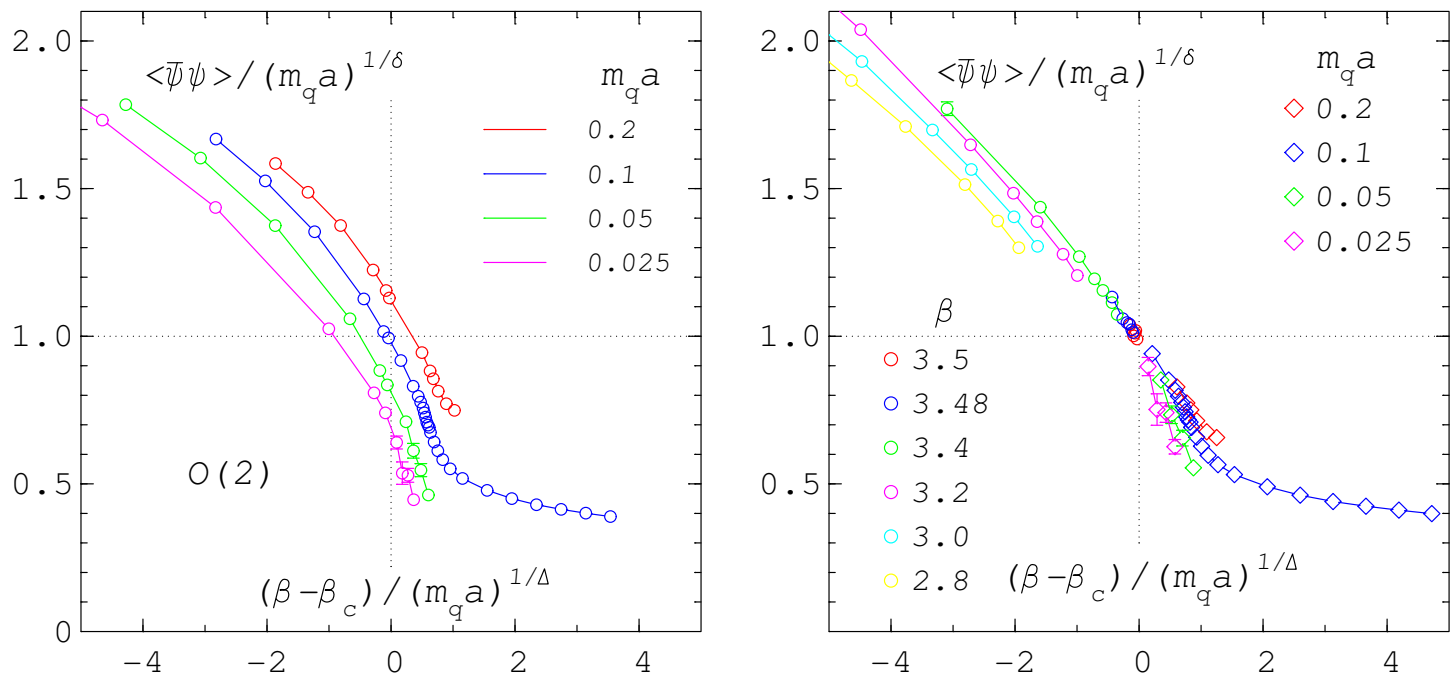

Figure 5: Test on scaling behaviour of $\langle\bar{\psi} \psi\rangle /\left(m_{q} a\right)^{1 / \delta}$ in $Q C D$ for $O(2)$ (left) and free (right) exponents.

\section{Summary}

In $a Q C D$ the deconfinement transition is first order and occurs below the second order chiral transition. The latter is located at $\beta_{c}=5.624(2)$. The scaling behaviour of $\langle\bar{\psi} \psi\rangle$ in the vicinity of $\beta_{c}$ is in full agreement with the $3 d O(2)$ universality class. The lattice data do not yet show scaling with exponents from the proposed continuum class[2]. In the region between the two phase transitions the quark mass dependence of the chiral condensate is as expected due to the existence of Goldstone modes like in $3 d O(N)$ spin models.

For $Q C D$ we find that the chiral condensate exhibits Goldstone effects below the chiral transition point as in $3 d O(N)$ spin models and like in $a Q C D$. The transition is of second order or a crossover, there is no sign of a first order behaviour. In the vicinity of $\beta_{c}$ the chiral condensate does not show the scaling behaviour of the $3 d O(2)$ or $O(4)$ universality classes, at best it is that of a class with exponents $\delta=2.3$ and $\beta_{m}=0.6$. This result could be a consequence of the coincidence of the deconfinement and the chiral transitions.

\section{References}

[1] F. Karsch and M. Lütgemeier, Deconfinement and chiral symmetry restoration in an SU(3) gauge theory with adjoint fermions, Nucl. Phys. B550 (1999) 449 [hep-lat/9812023].

[2] F. Basile, A. Pelissetto and E. Vicari, The finite-temperature chiral transition in QCD with adjoint fermions, JHEP 0502 (2005) 044 [hep-th/ 0 412026], and in the proceedings of this conference, PoS(LAT2005)199.

[3] J. Engels, S. Holtmann and T. Schulze, Scaling and Goldstone effects in a QCD with two flavours of adjoint quarks, Nucl. Phys. B724 (2005) 357 [hep-lat/ 0505008 ].

[4] U. M. Heller, F. Karsch and B. Sturm, Improved staggered fermion actions for QCD thermodynamics, Phys. Rev. D60 (1999) 114502 [hep-lat/9901010].

[5] F. Karsch, E. Laermann and A. Peikert, Quark mass and flavor dependence of the QCD phase transition, Nucl. Phys. B605 (2001) 579 [hep-lat/ 0012023$].$ 